\title{
Ventricular tachycardia due to infiltration of interatrial septum with gastric lymphoma
}

\author{
Ahmet Karagöz ${ }^{a *}$, Ender Örnek ${ }^{\mathrm{b}}$, Serkan Serdara ${ }^{\mathrm{a}}$, Emre Ertürk $^{\mathrm{a}}$, Begüm Yetiş Sayın ${ }^{\mathrm{a}}$ \\ ${ }^{a}$ Giresun Prof. Dr. A. İlhan Özdemir State Hospital, Giresun, Turkey \\ ${ }^{b}$ Numune Education and Research Hospital, Ankara, Turkey
}

ARTICLE INFO

\section{Article History}

Received $\quad 19 / 06 / 2012$

Accepted $\quad 27 / 06 / 2012$

\section{* Correspondence to:}

Ahmet Karagöz

Giresun Prof. Dr. A. İlhan Özdemir Devlet

Hastanesi, Kardiyoloji Bölümü,

Giresun, Türkiye

e-mail: drahmetkgz@hotmail.com

\section{Keywords:}

Interatrial septum

Metastasis

Ventricular tachycardia

\section{ABSTRACT}

Metastatic cardiac involvement occurs most often during the terminal stage of the malignant disease, associated with wide spread of the tumor, and it is generally diagnosed at autopsy. A 39-year old woman was taken to coronary intensive care unit with the diagnosis of ventricular tachycardia while she had been following in the medical oncology clinic for gastric lymphoma. Ventricular tachycardia was in polymorphic character. On the other hand, her first electrocardiogram in the oncology clinic was normal sinus rhythm. To rule out ischemia, coronary computed tomography (CT) angiography was done. All the coronary arteries were intact without any plaque formation. But thickening in the interatrial septum and superomedial and lateral walls of right atrium and increase in the soft tissue density were reported. The magnetic resonance imaging (MRI) reported a soft tissue lesion with $28 \times 37 \times 25 \mathrm{~mm}$ in size. The hyperintense lesion showed uptake of heterogenous contrast and expanded to both atrial cavities. The MRI report commented that it was reasonable to evaluate this view as the involvement of interatrial septum with metastasis of lymphoma especially when it was beared in mind that she had been under treatment of this disease. The appearance of a cardiac arrhythmia in the absence of known cardiac disease should raise the possibility of a cardiac metastasis in patients with malignant disease. So in patients with malign process, cardiac metastasis of the disease should be considered especially when patients present with cardiovascular symptoms.

J. Exp. Clin. Med., 2013; 30:79-80

\section{Introduction}

Clinically evident cardiac metastases from malignant neoplasms are uncommon, although they are more frequent than primary cardiac tumors (Loffler and Grille, 1990; McAllister, 1991). The most common involved primary tumors are carcinoma of the lung, carcinoma of the breast, lymphoma, and malignant melanoma (Abraham et al., 1990). Metastatic cardiac involvement occurs most often during the terminal stage of the malignant disease, associated with wide spread of the tumor, and it is generally diagnosed at autopsy. In this paper we report a case of an interatrial septal metastasis presenting with ventricular tachycardia and emphasize the diagnostic problems of secondary cardiac tumours, the symptoms of which are often unspecific and late.

\section{Case}

A 39-year old woman was taken to coronary intensive care unit with the diagnosis of ventricular tachycardia while she had been following in the medical oncology clinic for gastric lymphoma. She had no history of cardiovascular disease and did not define any cardiac symptom up to now. Ventricular tachycardia was ongoing when she was transfered to cardiology department and cordarone infusion was started promptly. No response to antiarrhythmic medication was seen, direct current cardioversion was perfomed two times and normal sinus rythm was achieved. Ventricular tachycardia was in polymorphic character (Fig. 1).

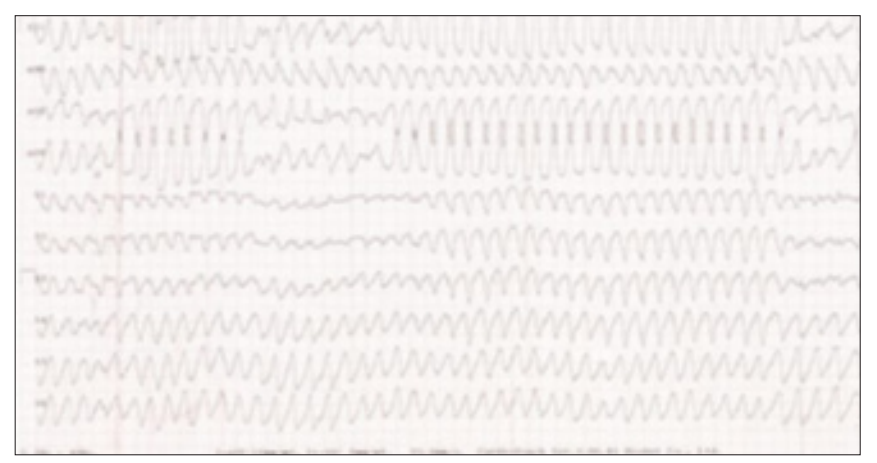

Fig. 1. Polymorphic ventricular tachycardia in the electrocardiogram of the patient. 
On the other hand, her first electrocardiogram in the oncology clinic was normal sinus rhythm (Fig. 2).

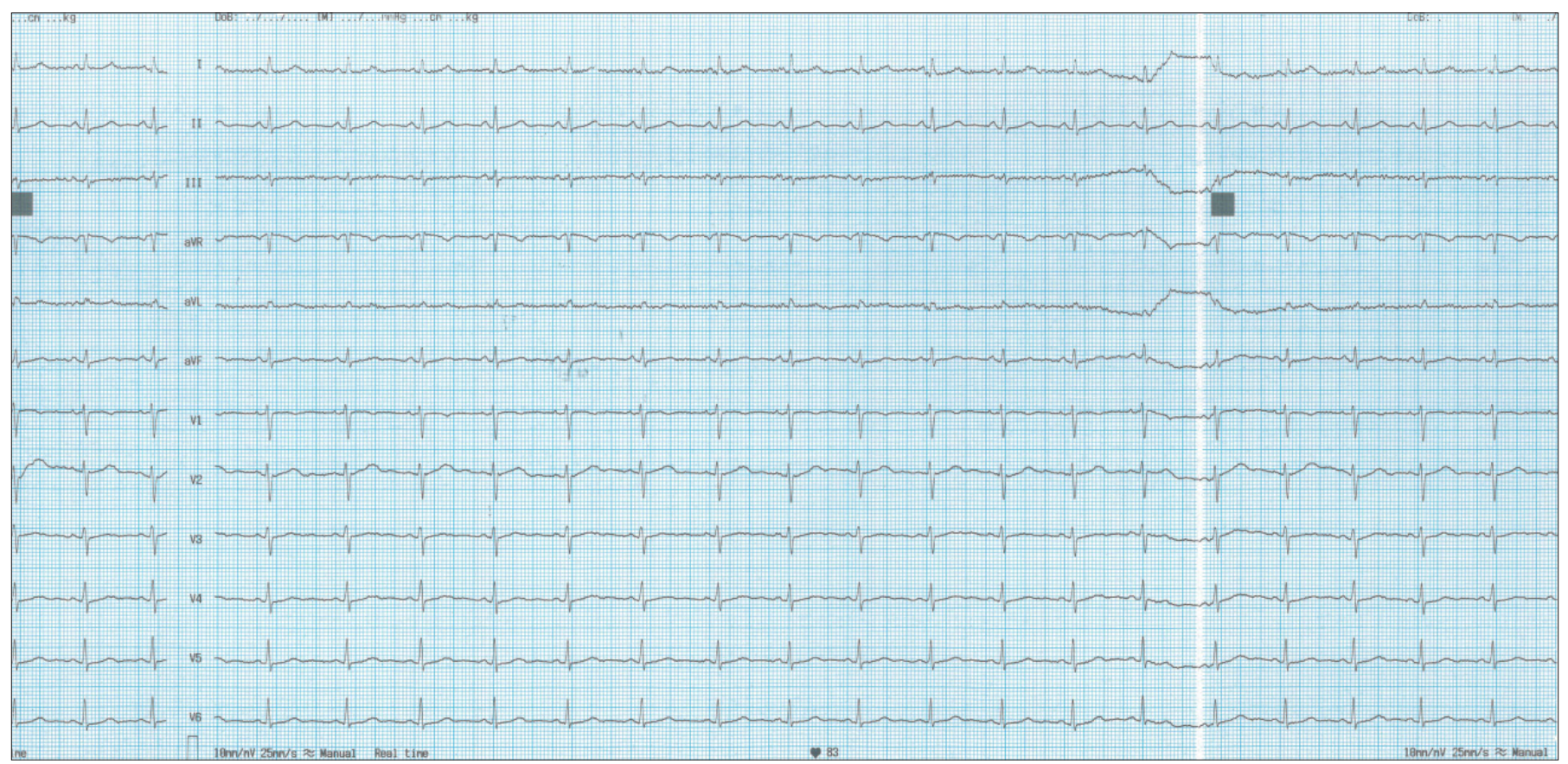

Fig. 2. Normal electrocardiogram of the patient before ventricular tachycardia.

To rule out ischemia, coronary computed tomography (CT) angiography was done. All of the coronary arteries were intact without any plaque formation. But thickening in the interatrial septum and superomedial and lateral walls of right atrium and increase in the soft tissue density were reported. Echocardiographic imaging also showed thickening of the interatrial septum with a left ventricular ejection fraction of $68 \%$ without any abnormality of other cardiac structures (Fig. 3).

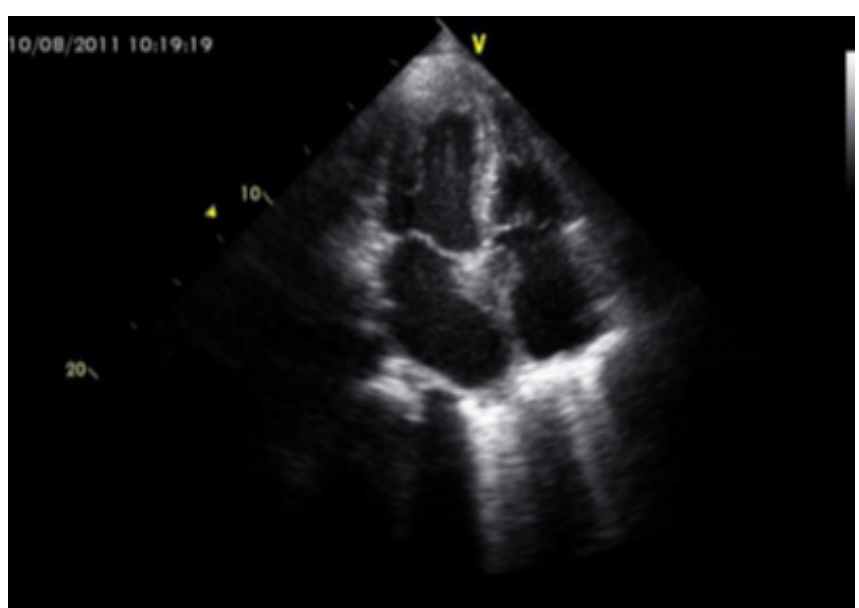

Fig. 3. Interatrial septal lesion in apical four chamber echocardiographic view.

Consequently it was rational to speculate that the interatrial septum may be infiltrated with metastasis of lymphoma.
Cardiac magnetic resonance was the choice of imaging modality to clarify the cause of polymorphic ventricular tachycardia in the absence of any other observable reason. The magnetic resonance imaging (MRI) reported a soft tissue lesion with $28 \times 37 \times 25 \mathrm{~mm}$ in size. The hyperintense lesion showed uptake of heterogenous contrast and expanded to both atrial cavities. The MRI report commented that it was reasonable to evaluate this view as the involvement of interatrial septum with metastasis of lymphoma especially when it was beared in mind that she had been under treatment of this disease. Therefore in the absence of any displayed reason for arrhytmia, we suggest that infiltration of atrial septum with lymphoma can cause ventricular tachycardia. It was also notable that although this process seems to be the cause of ventricular tachycardia, any abnormality was not seen in baseline electrocardiogram. The expected survival of the patient was longer than one year according to the oncologists. So, to prevent sudden cardiac death, implantable cardioverter defibrillator implantation was performed and the patient was transfered to the oncology clinic for continuation of chemotherapy.

\section{Conclusion}

The appearance of a cardiac arrhythmia in the absence of known cardiac disease should raise the possibility of a cardiac metastasis in patients with malignant disease. So in patients with malign process, cardiac metastasis of the disease should be considered especially when patients present with cardiovascular symptoms.

\section{REFERENCES}

Abraham, K.P., Reddy, V., Gattuso, P., 1990. Neoplasms metastatic to the heart: Review of 3314 consecutive autopsies. Am. J. Cardiovasc. Pathol. 3, 195-198.

Loffler, H., Grille, W., 1990. Classification of malignant cardiac tumors with respect to oncologic treatment. Cardiovasc. Thorac. Surg. 38, 173175.

McAllister, H.A. Jr., 1991. Tumors of the heart and pericardium in Cardiovascular Pathology, Edited by MD Silver, New York, Churchill Livingstone, pp. 1297-1334. 